\title{
The Study of the Three-Dimensional Meshless Solver Based on AUSM+-up and MUSCL Scheme
}

\author{
Yuanding Wang ${ }^{1, a}$, Xiaowei Cai ${ }^{2, b}$, Mu Zhang ${ }^{3, c}$, Xinjian Ma ${ }^{4, d}$, Dengfeng Ren ${ }^{1, e}$, \\ Junjie $\operatorname{Tan}^{1, f}$
}

1 School of Energy and Power Engineering, Nanjing University of Science and Technology, Nanjing, China

2 China Ship Scientific Research Center, National Key Laboratory of Science and Technology on Hydrodynamic, Wuxi, China

3 China Academy of Launch Vehicle Technology, Beijing, China

4 Shanghai Xinli Power Equipment Research Institute, Shanghai, China

awyd19870328@163.com, bxiaocai2601@163.com, nanjingzhangmo@163.com, dmaxinjian1981@yahoo.com.cn, ${ }^{\mathrm{e}}$ rendengfeng@njust.edu.cn, ddxyjx@mail.njust.edu.cn

Keywords: Meshless method, AUSM+-up , MUSCL Scheme, Euler equation, Least square

Abstract: The meshless method does not need the mesh cells to divide the computational domain, but use the 'clouds of points' to replace the function of mesh cells. For this reason, the meshfree method can bring the advantage of flexible point distribution to divide the computational domain. In the present paper, an efficient and accurate 3D least square meshless algorithm is shown to solve the Euler equations based on the AUSM+-up and MUSCL scheme. The explicit three-stage Strong Stability Preserving (SSP) Runge-Kutta method is chosen to advance the time evolution. The spatial derivatives are fitted by the least square method based on the cloud of point. The explicit expressions of the spatial derivatives are given in the present work. In order to investigate the robustness and accuracy of the present 3D meshless method, both transonic flow and supersonic flow are simulated. The results obtained show a good agreement with the exact solution or the experiment results.

\section{Introduction}

With the development of computer technology, Computational Fluid Dynamic (CFD) is playing an more and more important role in the practical engineering applications. In most of these applications, the geometrical configurations are very complex. It is difficult to use the conventional CFD methods, such as Finite volume method, Finite difference method and Finite element method to solve these problems. Especially for the complex unsteady flows with moving boundary, sometimes it is even impossible to achieve for these methods. The main reason is the grid generation, which is one of the major challenges in solving either Euler or Navier-Stokes equations over the complex geometrical configurations.

The meshless ('meshfree', 'gridless', or 'gridfree') method was first formally proposed by Lucy [1], which was successfully applied in the field of astrophysics. The meshless method only needs the nodes information, which can be called 'cloud of points', and does not require that the nodes be connected to form a mesh cell as is necessary in conventional mesh-based method. So the meshless method can bring the advantage of flexible point distribution. The most notable work for introducing the meshfree method into the Computational Fluid Dynamics (CFD) field was done first by Batina in 1992 [2], who developed an explicit least square meshfree algorithm based on the minimal surface approximation and used this kind of meshfree method to solve the compressible flows with shocks. From then on, a lot of meaningful work was done for meshless method in CFD (Morinishi[3], Cai et al[4], Katz[5], Chen and Shu[6]).

In the present paper, an efficient and accurate 3D least square meshless algorithm is shown to solve the Euler equations. The convection fluxes are calculated by the AUSM+-up scheme. And the Monotonic Upstream-Centered Scheme for Conservation Laws (MUSCL)-type approach with Van Albada limiter is used to achieve a high order scheme. The spatial derivatives is fitted by the least 
square method based on the cloud of point. The explicit expressions of the spatial derivatives are given in the present work. Some classical test cases are calculated to verify the robustness and accuracy of the present 3D meshless method. The simulation results show a good agreement with the experiment results and other numerical simulation results. Overall, simulation results demonstrate the validity and practicality of the present 3D method.

\section{Governing equations and numerical algorithm}

The three-dimensional, compressible Euler equations to be solved in the present paper can be expressed in the following form:

$$
\frac{\partial Q}{\partial t}+\frac{\partial F}{\partial x}+\frac{\partial G}{\partial y}+\frac{\partial H}{\partial z}=0
$$

Where

$$
Q=\left(\begin{array}{c}
\rho \\
\rho u \\
\rho v \\
\rho w \\
\rho E
\end{array}\right), F=\left(\begin{array}{c}
\rho u \\
\rho u^{2}+p \\
\rho v u \\
\rho w u \\
u(\rho E+p)
\end{array}\right), G=\left(\begin{array}{c}
\rho v \\
\rho u v \\
\rho v^{2}+p \\
\rho w v \\
v(\rho E+p)
\end{array}\right), H=\left(\begin{array}{c}
\rho w \\
\rho u w \\
\rho v w \\
\rho w^{2}+p \\
w(\rho E+p)
\end{array}\right)
$$

In equation 2, $u, v$ and $w$ represent velocity components in the $x, y$ and $\mathrm{z}$ directions. $\rho, e$ and $p$ are the density, total energy and pressure, respectively. The total energy can be calculated by :

$$
e=\frac{p}{\rho(\gamma-1)}+\frac{\left(u^{2}+v^{2}+w^{2}\right)}{2}
$$

Assuming that the fluid, air, is prefect gas, then the pressure can be calculated by the perfect gas equation of state

$$
p=(\gamma-1)\left[\rho e-\frac{\rho}{2}\left(u^{2}+v^{2}+w^{2}\right)\right]
$$

Where $\gamma$ denotes the ratio of specific heats of fluid and typically taken as 1.4 for air.

\section{Numerical Algorithm}

Spatial discretization. The least square meshless method only requires the information of the surrounding points at any given point, which is called "clouds of points", to solve the spatial derivatives. Fig. 1 shows the illustration of cloud of points and fluxes split.

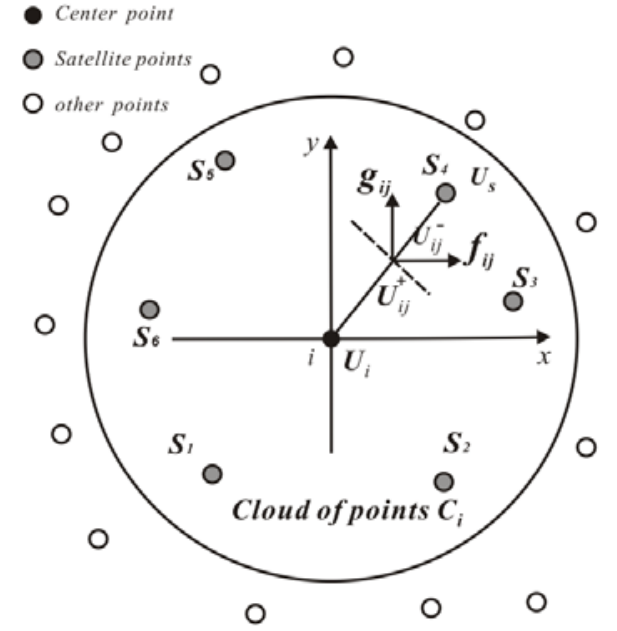

Fig. 1. The illustration of cloud of points and fluxes split. 
The spatial derivatives of point $i$ is calculated by the weighted least square method, which is based on Taylor formulation in the present paper. Assuming that arbitrary function $\phi(x, y, z)$ represented by "cloud of point" $C_{i}$, and can be written as:

$$
\phi_{j}=\phi_{i}+\left.\left(x_{j}-x_{i}\right) \frac{\partial \phi}{\partial x}\right|_{i}+\left.\left(y_{j}-y_{i}\right) \frac{\partial \phi}{\partial y}\right|_{i}+\left.\left(z_{j}-z_{i}\right) \frac{\partial \phi}{\partial z}\right|_{i}+O\left(h^{2}\right), \quad j \in C_{i}
$$

The spatial derivatives are determined by using a weighted least square approximation:

$$
\min \sum_{j \in C_{i}} w_{i j}\left(\Delta \phi_{i j}-\left.\Delta x_{i j} \frac{\partial \phi}{\partial x}\right|_{i}-\left.\Delta y_{i j} \frac{\partial \phi}{\partial y}\right|_{i}-\left.\Delta z_{i j} \frac{\partial \phi}{\partial z}\right|_{i}\right)^{2}
$$

Where $\Delta \phi_{i j}=\phi_{j}-\phi_{i}, \Delta x_{i j}=x_{j}-x_{i}, \Delta y_{i j}=y_{j}-y_{i}, \Delta z_{i j}=z_{j}-z_{i}$ and $w_{i j}=1 /\left(\Delta x_{i j}{ }^{2}+\Delta y_{i j}{ }^{2}+\Delta \mathrm{z}_{i j}{ }^{2}\right)^{1 / 2}$. The solution of this minimization problem leads to a matrix equation according to the principle of the least square scheme:

$$
\left[\begin{array}{ccc}
\sum_{j \in C_{i}} w_{i j} \Delta x_{i j}{ }^{2} & \sum_{j \in C_{i}} w_{i j} \Delta x_{i j} \Delta y_{i j} & \sum_{j \in C_{i}} w_{i j} \Delta x_{i j} \Delta z_{i j} \\
\sum_{j \in C_{i}} w_{i j} \Delta x_{i j} \Delta y_{i j} & \sum_{j \in C_{i}} w_{i j} \Delta y_{i j}{ }^{2} & \sum_{j \in C_{i}} w_{i j} \Delta y_{i j} \Delta z_{i j} \\
\sum_{j \in C_{i}} w_{i j} \Delta x_{i j} \Delta z_{i j} & \sum_{j \in C_{i}} w_{i j} \Delta y_{i j} \Delta z_{i j} & \sum_{j \in C_{i}} w_{i j} \Delta z_{i j}{ }^{2}
\end{array}\right]\left[\begin{array}{l}
\left.\frac{\partial \phi}{\partial x}\right|_{i} \\
\left.\frac{\partial \phi}{\partial y}\right|_{i} \\
\left.\frac{\partial \phi}{\partial z}\right|_{i}
\end{array}\right]=\left[\begin{array}{l}
\sum_{j \in C_{i}} w_{i j} \Delta x_{i j}\left(\phi_{i j}-\phi_{i}\right) \\
\sum_{j \in C_{i}} w_{i j} \Delta y_{i j}\left(\phi_{i j}-\phi_{i}\right) \\
\sum_{j \in C_{i}} w_{i j} \Delta z_{i j}\left(\phi_{i j}-\phi_{i}\right)
\end{array}\right]
$$

The solution of the derivatives can be simply written as the following linear approximation when solving the above equation explicitly:

$$
\left.\frac{\partial \phi}{\partial x}\right|_{i}=\alpha \sum_{j \in C_{i}} a_{i j}\left(\phi_{j}-\phi_{i}\right),\left.\frac{\partial \phi}{\partial y}\right|_{i}=\sum_{j \in C_{i}} \beta\left(\phi_{j}-\phi_{i}\right),\left.\frac{\partial \phi}{\partial z}\right|_{i}=\sum_{j \in C_{i}} \gamma_{i j}\left(\phi_{j}-\phi_{i}\right)
$$

Where the coefficients $\alpha_{i j}$, $\beta_{i j}$ and $\gamma_{i j}$ are defined as the following form:

$$
\left\{\begin{array} { l } 
{ \alpha _ { i j } = a _ { i j , 1 } - \frac { l _ { 1 2 } } { l _ { 1 1 } } a _ { i j , 2 } + \frac { l _ { 1 2 } l _ { 2 3 } - l _ { 1 3 } l _ { 2 2 } } { l _ { 1 1 } l _ { 2 2 } } a _ { i j , 3 } } \\
{ \beta _ { i j } = a _ { i j , 2 } - \frac { l _ { 2 3 } } { l _ { 2 2 } } a _ { i j , 3 } } \\
{ \gamma _ { i j } = a _ { i j , 3 } }
\end{array} \text { and } \left\{\begin{array}{l}
a_{i j, 1}=\frac{\Delta x_{i j}}{l_{11}^{2}} \\
a_{i j, 2}=\frac{1}{l_{22}{ }^{2}}\left(\Delta y_{i j}-\frac{l_{12}}{l_{11}} \Delta x_{i j}\right) \\
a_{i j, 3}=\frac{1}{l_{33}{ }^{2}}\left(\Delta z_{i j}-\frac{l_{23}}{l_{22}} \Delta y_{i j}+\frac{l_{12} l_{23}-l_{13} l_{22}}{l_{11} l_{22}} \Delta x_{i j}\right)
\end{array}\right.\right.
$$

Where

$$
\left\{\begin{array}{l}
l_{11}=\sqrt{\sum_{s=1}^{N} w_{i j}\left(\Delta x_{i j}\right)^{2}}, l_{12}=\frac{1}{l_{11}} \sum_{s=1}^{N} w_{i j} \Delta x_{i j} \Delta y_{i j}, l_{22}=\sqrt{\sum_{s=1}^{N} w_{i j}\left(\Delta y_{i j}\right)^{2}-l_{12}^{2}} \\
l_{13}=\frac{1}{l_{11}} \sum_{s=1}^{N} w_{i j} \Delta x_{i j} \Delta z_{i j}, l_{23}=\frac{1}{l_{22}}\left(\sum_{s=1}^{N} w_{i j} \Delta y_{i j} \Delta z_{i j}-\frac{l_{12}}{l_{11}} \sum_{s=1}^{N} w_{i j} \Delta x_{i j} \Delta z_{i j}\right) \\
l_{33}=\sqrt{\sum_{s=1}^{N} w_{i j}\left(\Delta z_{i j}\right)^{2}-\left(\mathrm{l}_{13}{ }^{2}+l_{23}{ }^{2}\right)}
\end{array}\right.
$$

Finally, a semi-discrete form of the 3D Euler equations can be written as follows:

$$
\frac{\partial Q_{i}}{\partial t}+\sum_{j \in C_{i}}\left\{\left[\alpha_{i j}\left(\mathrm{~F}_{i j}-F_{i}\right)+\beta_{i j}\left(G_{i j}-G_{i}\right)+\gamma_{i j}\left(H_{i j}-H_{i}\right)\right]\right\}=0
$$

Inviscid fluxes discretization. According to the work done by Sridar and Balakrishnan[7], a fictitious interface $J$ is built at the midpoint of ray $i j$, as shown in Fig. 1. The AUSM+-up scheme is used to compute the inviscid fluxes as the following form:

$$
F_{1 / 2}=\frac{1}{2} a_{1 / 2}\left[M_{1 / 2}\left(\phi_{J}+\phi_{J+1}\right)-\left|M_{1 / 2}\right|\left(\phi_{J+1}-\phi_{J}\right)\right]+p_{1 / 2}
$$


More details can be found in the work done by Liou[8] and Cai[4].

Higher-order interpolation. In order to achieve a high order scheme, the Monotonic Upstream-Centered Scheme for Conservation Laws (MUSCL) reconstruction approach with Van Albada limiter is adopted to interpolate the primary variables onto the two sides of the fictitious interface in the present work. The MUSCL reconstruction scheme with a Van Albada limiter can be described as follows:

$$
u_{i j}^{+}=u_{i}+\frac{s_{i}}{4}\left[\left(1-k s_{i}\right) \Delta_{i j}^{-}+\left(1+k s_{i}\right)\left(u_{j}-u_{i}\right)\right] ; u_{i j}^{-}=u_{j}-\frac{s_{j}}{4}\left[\left(1-k s_{j}\right) \Delta_{i j}^{+}+\left(1+k s_{j}\right)\left(u_{j}-u_{i}\right)\right]
$$

where $\Delta_{i j}^{-}=2 \Delta \vec{r}_{i j} \bullet \nabla u_{i}-\left(u_{j}-u_{i}\right)$ and $\Delta_{i j}^{+}=2 \Delta \vec{r}_{i j} \bullet \nabla u_{j}-\left(u_{j}-u_{i}\right)$. Here, $\Delta \vec{r}_{i j}$ is the vector pointing from node $i$ to $j$. The gradient $\nabla u$ is calculated by equation 6 . The limiter $s$ is defined as:

$$
s_{i}=\max \left[0, \frac{2 \Delta_{i j}^{-}\left(u_{j}-u_{i}\right)+\varepsilon}{\left(\Delta_{i j}^{-}\right)^{2}+\left(u_{j}-u_{i}\right)^{2}+\varepsilon}\right] ; s_{j}=\max \left[0, \frac{2 \Delta_{i j}^{+}\left(u_{j}-u_{i}\right)+\varepsilon}{\left(\Delta_{i j}^{+}\right)^{2}+\left(u_{j}-u_{i}\right)^{2}+\varepsilon}\right]
$$

Where $\varepsilon$ is a very small positive number for preventing null division, and $\varepsilon=10^{-12}$ is used in this study. The parameter $k=1 / 3$ is set in the present work.

Temporal discretization. In the present paper, an explicit three-stage Strong Stability Preserving (SSP) Runge-Kutta method is chosen to advance the time evolution.Assuming that the variable $Q_{i}^{n}$ is known at time $t^{n}$, the solution is advanced over a time step $\Delta t$ from $Q_{i}^{n}$ to $Q_{i}^{n+1}$ at time $t^{n+1}$ by the following process:

$$
Q_{i}^{(1)}=Q_{i}^{n}-\Delta t R_{i}\left(Q_{i}^{n}\right) ; Q_{i}^{(2)}=\frac{3}{4} Q_{i}^{n}+\frac{1}{4}\left[Q_{i}^{(1)}-\Delta t R_{i}\left(Q_{i}^{(1)}\right)\right] ; Q_{i}^{n+1}=\frac{1}{3} Q_{i}^{n}+\frac{2}{3}\left[Q_{i}^{(2)}-\Delta t R_{i}\left(Q_{i}^{(2)}\right)\right]
$$

Where $\Delta t$ represents the time step. In order to accelerate convergence, the local time step and implicit residual smoothing technology is used in the present paper.

\section{Numerical simulation results and analysis}

Supersonic flow around sphere. The point distribution around sphere is shown in Fig. 2. Total 386188 points are placed on the computational domain. The radius of the sphere is $r=1$ and the flow Mach number is $M a=3.0$. A bow shock will appear in front of the sphere when the supersonic flow past over the sphere. Billig [9] gives the formula of the distance from the bow shock to the 3D sphere stagnation point according to a lot of theoretical derivation and analysis of experiment data:

$$
\frac{\Delta}{r}=0.143 \exp \left(\frac{3.24}{M a_{\infty}{ }^{2}}\right)
$$

So the analytical distance in the condition of $M a=3.0$ and $r=1$ is $\Delta \approx 0.205$. The numerical simulation result using the present $3 \mathrm{D}$ meshless method is $\Delta \approx 0.212$. The relative error is only $3.41 \%$.

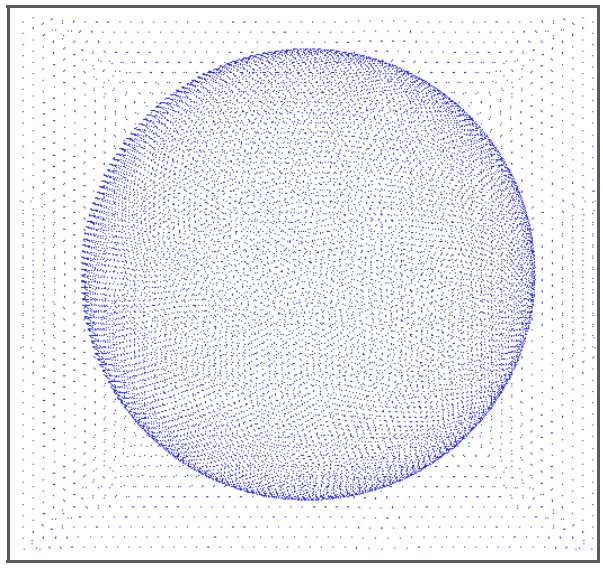

Fig. 2. Point distribution

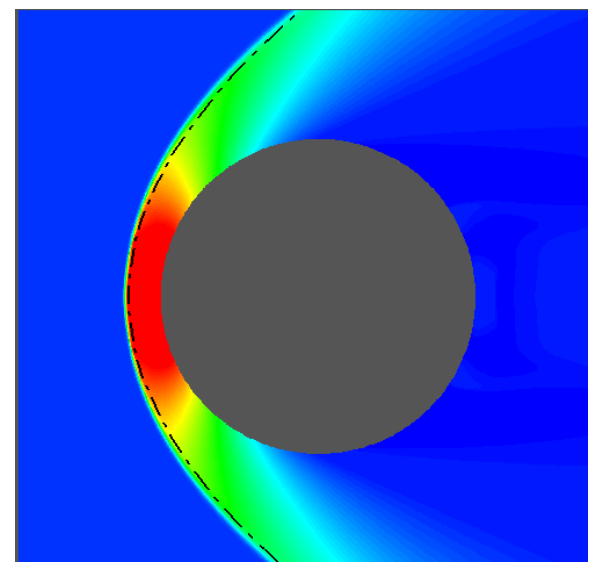

Fig.3. The bow shock shape comparison 
Billig believed that the detached bow shock shape can be described by a hyperbolic equation. Fig. 3 shows the density contours on the plane of $\mathrm{z}=0$, and the black dashed line is the theoretical curve of the detached bow shock given by Billig. The detached bow shock is clearly captured by the present 3D meshless method, and the calculated shock shape and position agree well with the theoretical curve.

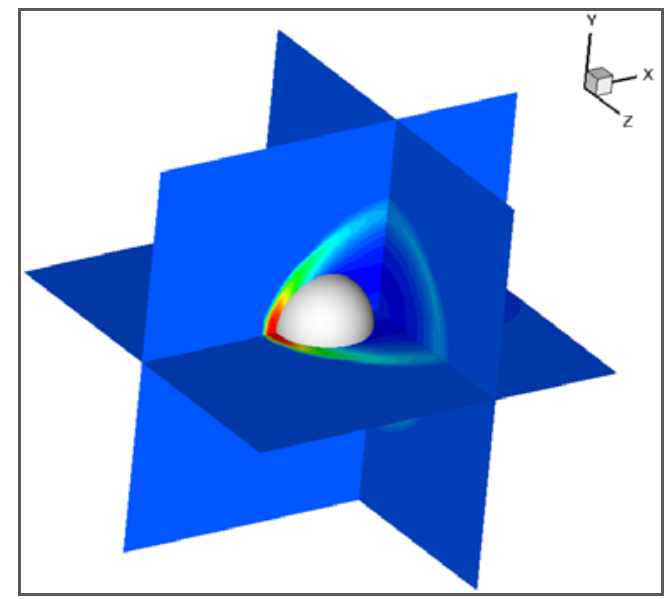

(a) pressure contours

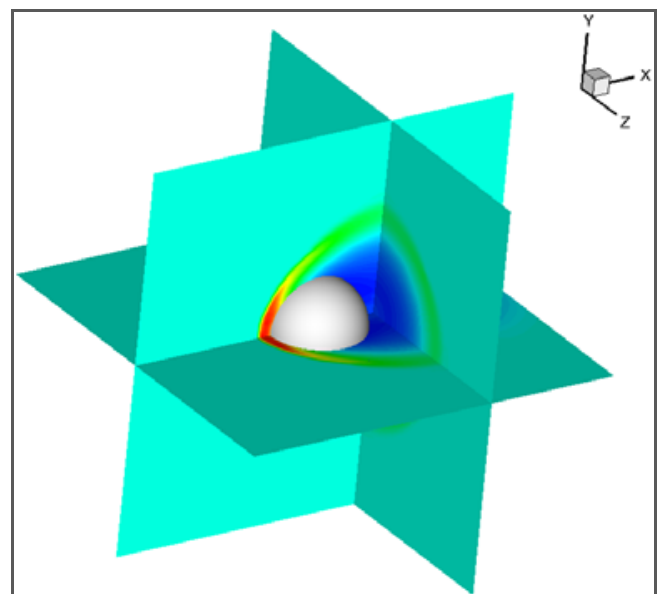

(b) density contours

Fig. 4. Color contours of the supersonic flow around sphere

The color contours of the flow field are shown in Fig. 4.The Convergence history is given in Fig. 5(a). Generally, the convergent results are obtained after 2000 iterations.

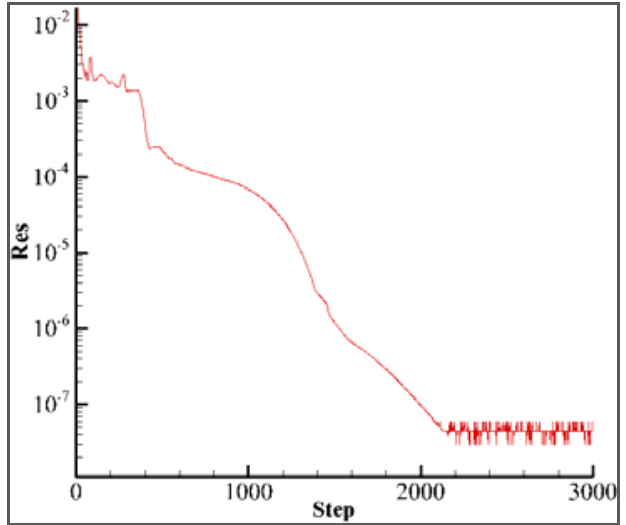

(a) For sphere case

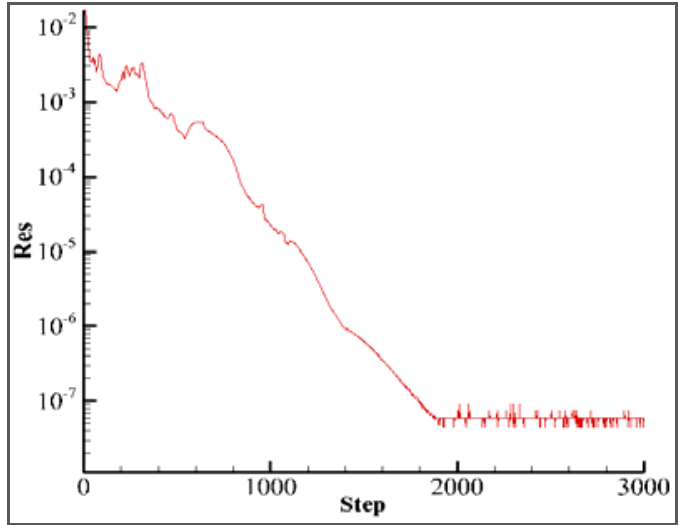

(b) For ONERA M6 wing case

Fig.5. Convergence history

Transonic flow around ONERA M6 wing. The second test case is the transonic flow over the ONERA M6 wing with the Mach number and angle of attack of $M a=0.8395$ and $\alpha=3.06$, the sideslip angle is 0 . A series of the complex flow phenomena, such as the $\lambda$ wave, will appear in the flow field when the transonic flow past the wing, even though the geometry configuration is not complex. The point distribution is shown in Fig. 6 and total 435918 points is placed on the computational domain. 


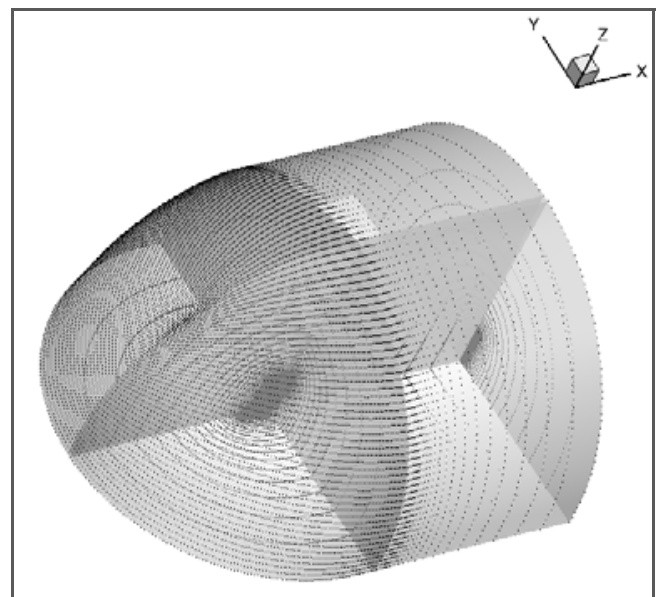

(a) all domain

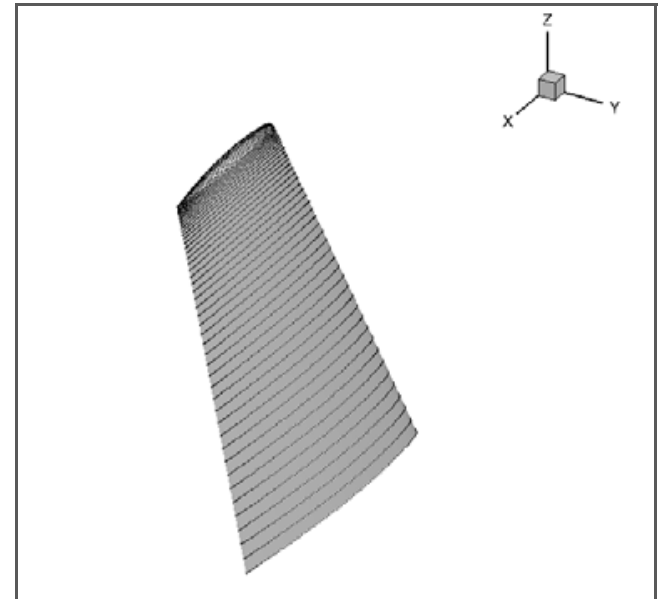

(b) surface of the wing

Fig. 6. The point distribution of the computational domain

The pressure contours of the wing surface are shown in Fig. 7(a), which gives the information that the $\lambda$ wave is clearly captured. The Mach number contours in different sections are shown in Fig.7(b). The convergence of the CL and CD are shown in Fig.8 and the convergence of the residual is shown in Fig. 5(b). And the convergent results are obtained after 2000 iterations.
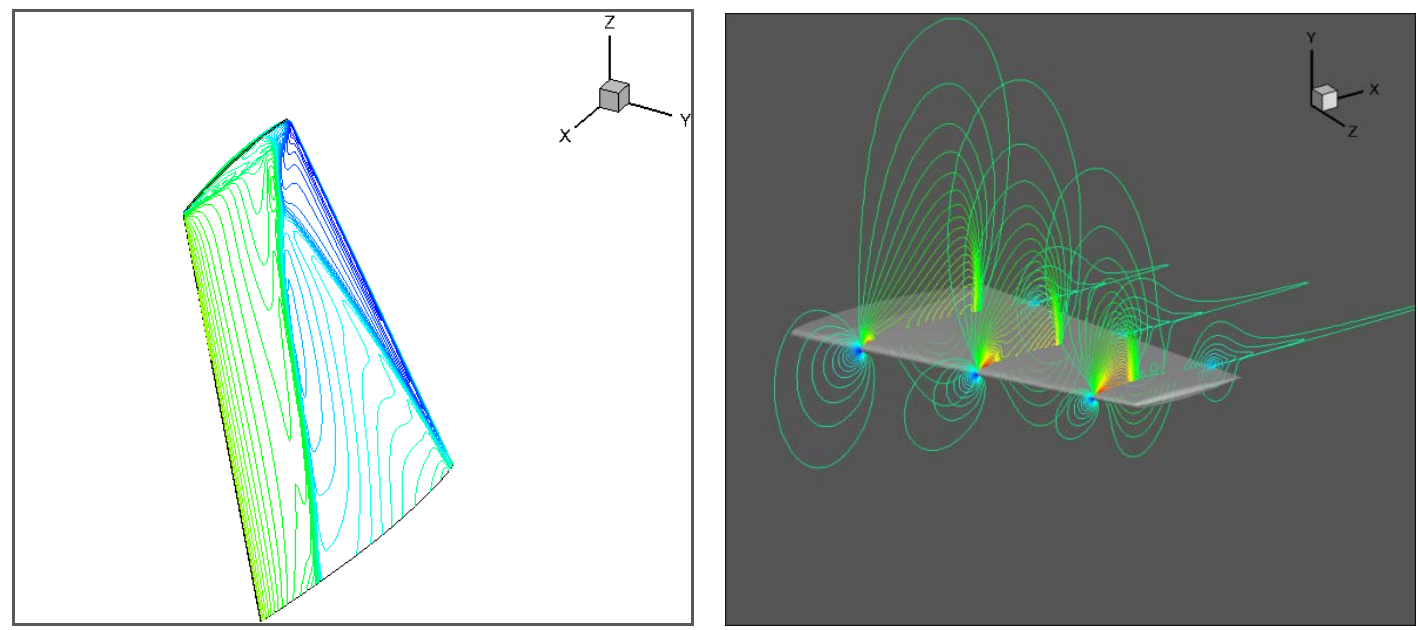

(a) pressure contours $(\lambda$ wave)

(b) Mach number contours in different sections

Fig. 7. Pressure contours and Mach contours

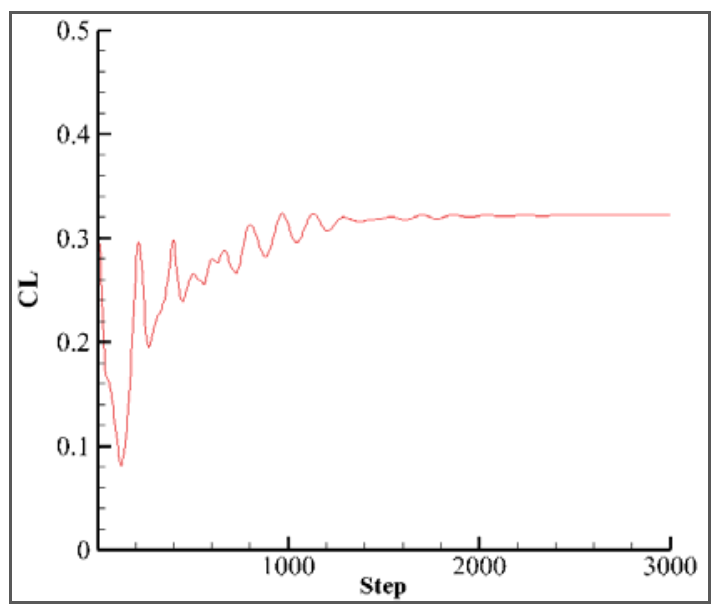

(a) CL

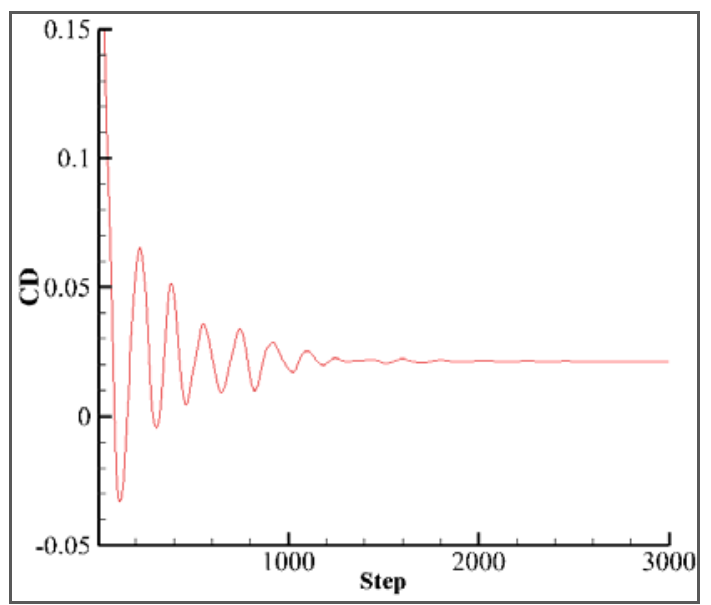

(b) CD

Fig. 8 The convergence of CL and CD 
In order to further confirm the accuracy and effectiveness of the present 3D meshless algorithm, the pressure coefficient at different place along the spanwise direction are compared with the experimental data [10], as shown in Fig. 9. It can be seen from the Figures that most of the simulated results are in good agreement with the experimental data. Only the results in the local area with the shock discontinuity is slightly different from the experimental data. The main reason maybe that the experiment data is obtained with the viscous flow, but the present numerical method is for Euler equations and does not consider the viscous effect. Overall, simulation results demonstrate that the proposed meshfless numerical algorithm is accurate and capable of simulating 3D flows.

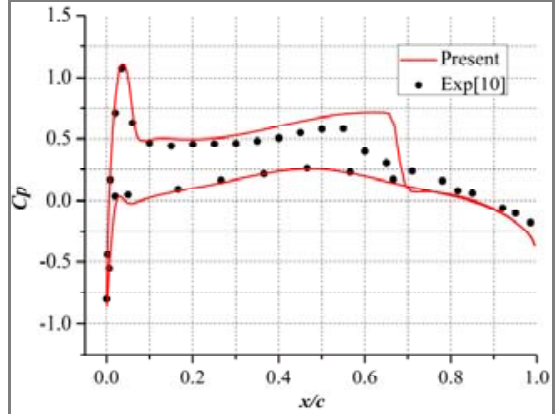

(a) $\eta=0.20$

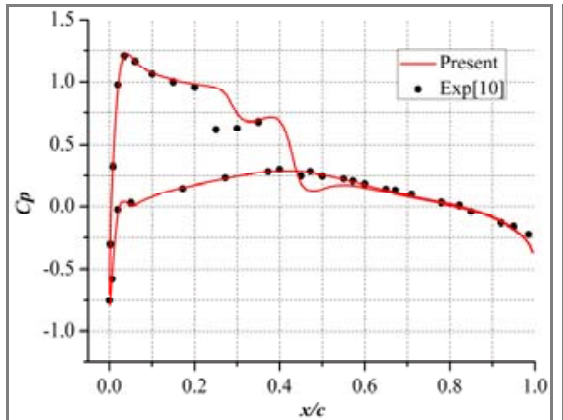

(d) $\eta=0.80$

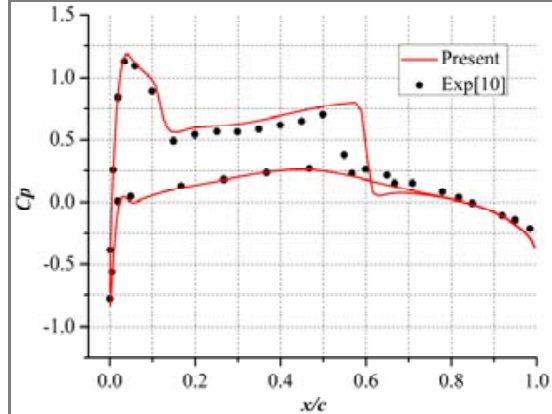

(b) $\eta=0.44$

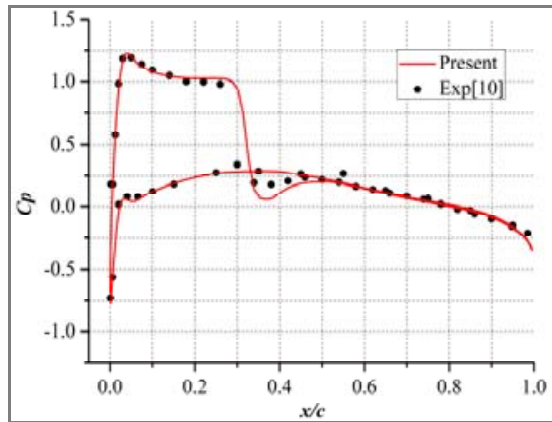

(e) $\eta=0.90$

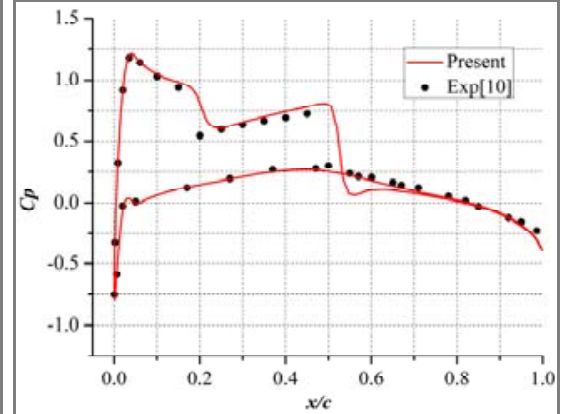

(c) $\eta=0.65$

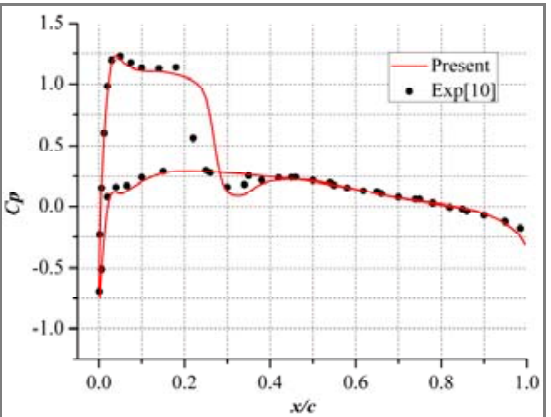

(f) $\eta=0.95$

Fig. 9. The pressure coefficient at different place along the spanwise direction

\section{Conclusion}

The present paper presents a 3D explicit meshless method for solving the compressible Euler equations. The AUSM+-up scheme with MUSCL approach is used to calculate the convection fluxes. Some standard test cases are simulated to verify the robustness and accuracy of the present 3D meshless method. The simulation results obtained by the present 3D meshless method are compared with the exact results and the experimental results. The good agreement with the reference data validates the accuracy and efficiency of the proposed 3D least square meshless method.

\section{Acknowledgements}

This research has been supported by the [National Natural Science Foundation of China] under Grant [number 11072114]; and [Science and Technology Innovation Foundation of Shanghai Aerospace] under Grant [number SAST201365].

\section{References}

[1] Lucy L.B. A numerical approach to the testing of the fission hypothesis. The Astronomical Journal, 1977, 82:1013-1024.

[2] Batina JT. A gridless Euler/Navier-Stokes solution algorithm for complex two-dimensional applications. NASA-TM-107631, June 1992. 
[3] Morinishi K. An implicit gridless type solver for the Navier-Stokes equations. Computational Fluid Dynamics Journal, 2001(Special Issue):551-560.

[4] Cai X.W., Tan J.J., Ma X.J., et al. Application of hybrid Cartesian grid and gridless approach to moving boundary flow problems. International Journal For Numerical Methods In Fluids. 2013, 72: 994-1013.

[5] Katz, A. and Jameson, A. Edge-based Meshless Methods for Compressible Viscous Flow with Applications to Overset Grids, AIAA paper 2008-3989, AIAA 38th Fluid Dynamics Conference, Seattle, WA, June 2008.

[6] Chen H.Q., Shu C. An Efficient Implicit Mesh-Free Method To Solve Two-Dimensional Compressible Euler Equations. International Journal of Modern Physics C. 2005,16:439- 454.

[7] Sridar D., Balakrishnan N. An upwind finite difference scheme for meshless solvers. Journal of Computational Physics, 2003, 189: 1-29.

[8] Liou M S. A sequel to AUSM, Part II: AUSM+-up for all speeds. Journal of Computational Physics, 2006, 214:137-170.

[9] Billig FS. Shock-wave shapes around spherical- and cylindrical-nosed bodies. Journal of Spacecraft and Rockets, 1967, 6: 822-823.

[10] Schmitt V, Charpin F. Pressure Distribution on the ONERA M6 Wing at Transonic Mach Numbers[R]. Appendix B1 in AGARD-AR-138, Experimental Data Base for Computer Program Assessment, 1979. 